\title{
Tail Asymptotic Expansions for L-Statistics
}

\author{
Enkelejd Hashorva* \& Chengxiu Ling* \& Zuoxiang Peng ${ }^{\dagger}$ \\ *Department of Actuarial Science, Faculty of Business and Economics (HEC Lausanne), \\ University of Lausanne, 1015 Lausanne, Switzerland. \\ ${ }^{\dagger}$ School of Mathematics and Statistics, Southwest University, 400715 Chongqing, China
}

\begin{abstract}
In this paper, we derive higher-order expansions of $L$-statistics of independent risks $X_{1}, \ldots, X_{n}$ under conditions on the underlying distribution function $F$. The new results are applied to derive the asymptotic expansions of ratios of two kinds of risk measures, stop-loss premium and excess return on capital, respectively.
\end{abstract}

Keywords: Smoothly varying condition; Second-order regular variation; Tail asymptotics; Value-at-Risk; Conditional tail expectation; Largest claims reinsurance; Ratio of risk measure; Excess return on capital.

MR(2010): Subject Classification 60E05, 60F99.

\section{Introduction}

Let positive random variables $X_{1}, X_{2}, \ldots, X_{n}$ denote $n$ risks and denote $X_{1, n} \leq \ldots \leq X_{n, n}$ their order statistics. Define

$$
S_{n}(\mathbf{c})=c_{1} X_{n, n}+c_{2} X_{n-1, n}+\cdots+c_{n} X_{1, n},
$$

with $\mathbf{c}=\left(c_{1}, c_{2}, \ldots, c_{n}\right) \in(0, \infty)^{2} \times[0, \infty)^{n-2}$; investigation of the random variable $S_{n}(\mathbf{c})$, which is an $L$ statistics is of interest in statistics, applied probability, actuarial mathematics, risk management and many other fields. Typically, the properties of $S_{n}(\mathbf{c})$ are derived when $n$ becomes large, i.e., $n \rightarrow \infty$, see Beirlant and Teugels [8, Ladoucette and Teugels [19, Ladoucette and Teugels 20, Ladoucette and Teugels [21] which also present several financial and insurance applications.

In other applications, for instance when modelling the financial losses of $n$ portfolios, it is not possible to change the number of portfolios under investigation, and therefore of interest is the tail asymptotic behaviour of $S_{n}$ (c) for each fixed $n$. The recent contribution Asimit et al. [5] (see also Asimit and Badescu [2], Asimit and Jones [3, Asimit and Jones [4) shows that under weak asymptotic conditions

$$
\mathbb{P}\left(S_{n}(\mathbf{c})>x\right) \sim \mathbb{P}\left(c_{1} X_{n, n}>x\right) \quad \text { as } \quad x \rightarrow \infty,
$$

which means that the maximum controls the asymptotic behaviour of the $L$-statistics $S_{n}(\mathbf{c})$. For applications, it is of interest to know the speed of convergence to 0 of $\Delta(x)=\mathbb{P}\left(S_{n}(\mathbf{c})>x\right)-\mathbb{P}\left(c_{1} X_{n, n}>x\right)$, i.e., how well the maximum risk controls the $L$-statistics $S_{n}(\mathbf{c})$. Since in many cases the tail asymptotics of $X_{n, n}$ might be unknown, it is of interest to derive higher-order asymptotic expansions for the tail of $S_{n}(\mathbf{c})$ in terms of tail asymptotics of $X_{i}$ 's. Clearly, when $c_{1}=\cdots=c_{n}=1$ we have $S_{n}(\mathbf{c})=\sum_{i=1}^{n} X_{i}=: S_{n}$; the second-order tail behavior of $S_{n}$ has been investigated under some smoothness conditions by Degen et al. [10, Mao et al. [23], Omey and Willekens [24. Further results on the higher-order tail asymptotics can be found in Albrecher et al. 1], Barbe and McCormick [6, Geluk et al. [14]. Results for the second-order tail asymptotics of $S_{n}$ under some second-order regular variation conditions are derived in Geluk et al. [13, Kortschak [17, Mao and Hu 22. even for dependent cases.

In this paper, we will first investigate the higher-order tail asymptotics of $S_{n}(\mathbf{c})$ under some smoothness condition for the iid $X_{i}$ 's, and then we derive the second-order tail asymptotics of $S_{n}(\mathbf{c})$ in the second-order

Work of E. Hashorva and C. Ling was supported from the Swiss National Science Foundation Grant 200021-140633/1 and the project RARE -318984 (an FP7 a Marie Curie IRSES Fellowship). Z. Peng was supported by the National Natural Science Foundation of China grant no.11171275 and the Natural Science Foundation Project of CQ no. cstc2012jjA00029. 
framework. Finally we apply our results to establish the following second-order approximations: ratio of tail Value-at-Risk (TVaR) and Value-at-Risk (VaR) and ratio of tail conditional tail expectation (TCTE) and conditional tail expectation (CTE); stop-loss premium and excess return on capital.

The contents of this paper are organized as follows. In Section 2 we recall the definitions of smoothness varying and the second-order regular variation, and give some useful lemmas. In Section 3 we present the higher-order asymptotic expansion and the second-order tail asymptotic expansion of $S_{n}(\mathbf{c})$ followed by a section dedicated to the second-order asymptotic expansion of two kinds of risk measures, stop-loss premium and excess return on capital. The proofs of all results are relegated to Section 6 .

\section{Preliminaries}

In the sequel, we will always consider independent risks $X_{i}$ 's with some common underling distribution function (df) $F$. We write $\bar{F}=1-F$ for the survival function of $F$ and $\mathbb{I}\{\cdot\}$ for the indicator function, and denote by $\lceil\alpha\rceil$ the smallest integer $l$ such that $\alpha \leq l$. In order to derive higher-order tail asymptotics of $S_{n}(\mathbf{c})$ we shall assume that $\bar{F}$ is a smoothly varying function, defined below as in Barbe and McCormick [7].

Definition 2.1. $\bar{F}$ is smoothly varying with index $-\alpha$ and order $m \in \mathbb{N}$, denoted by $\bar{F} \in \mathrm{SR}_{-\alpha, m}$ if $\bar{F}$ is eventually $m$ times continuously differentiable and $\bar{F}^{(m)}$ is regularly varying with index $-(\alpha+m)$, i.e., $\lim _{t \rightarrow \infty} \bar{F}^{(m)}(t x) / \bar{F}^{(m)}(t)=x^{-(\alpha+m)}$ for all $x>0$, denoted by $\bar{F}^{(m)} \in \mathrm{RV}_{-(\alpha+m)}$.

Next, we recall the definition of the second-order regular variation, see de Haan and Ferreira 9 .

Definition 2.2. $\bar{F}$ is said to be of second-order regular variation with parameters $\alpha \in \mathbb{R}$ and $\rho \leq 0$, denoted by $\bar{F} \in 2 \mathrm{RV}_{-\alpha, \rho}$, if there exists some function $A$ with constant sign near infinity satisfying $\lim _{t \rightarrow \infty} A(t)=0$ such that

$$
\lim _{t \rightarrow \infty} \frac{\bar{F}(t x) / \bar{F}(t)-x^{-\alpha}}{A(t)}=x^{-\alpha} \int_{1}^{x} u^{\rho-1} d u=: H_{-\alpha, \rho}(x)
$$

holds locally uniformly for all $x>0$.

In the literature, the function $A(\cdot)$, satisfying $\lim _{t \rightarrow \infty} A(t)=0$ and $|A| \in \mathrm{RV}_{\rho}$, is commonly referred to as the auxiliary function of $\bar{F}$. Obviously, equation (2.1) implies $\bar{F} \in \mathrm{RV}_{-\alpha}$ and the second-order parameter $\rho$ controls the convergence rate of $\bar{F}(t x) / \bar{F}(t)-x^{-\alpha}$. Several classes of parametric survival functions are shown to possess 2RV properties, see e.g., Hashorva et al. [15].

Remark 2.3. For the standard Pareto model $\bar{F}(x)=x^{-\alpha}$, the convergence of $\bar{F}(t x) / \bar{F}(t)$ is immediate, which is interpreted as $\rho=-\infty$ in (2.1). Some examples of Hall-class, absolute student $t$ distribution and $g$-and- $h$ distribution possessing 2RV are given in Section 5 for $\rho<0$ and $\rho=0$.

Hereafter we shall use some specific notation. Define

$$
S_{n-1}(\mathbf{c})=c_{2} X_{n-1, n-1}+\cdots+c_{n} X_{1, n-1}, \quad S_{(n)}(\mathbf{c})=S_{n}(\mathbf{c})-c_{1} X_{n, n}
$$

and let $F_{n}$ denote the df of $S_{n-1}(\mathbf{c})$. Without loss of generality, assume that the constant $\mathbf{c}$ is such that $\mathbf{c}=\left(1, c_{2}, \ldots, c_{n}\right) \in\{1\} \times(0, \infty) \times[0, \infty)^{n-2}$, and set

$$
\widetilde{\mathbf{c}}=\frac{c_{2}}{1+c_{2}} .
$$

In order to derive higher-order behavior of $S_{n}(\mathbf{c})$, we need some auxiliary results. The first lemma generalizes Lemma 3.1 in Albrecher et al. [1].

Lemma 2.4. If $\bar{F} \in \mathrm{RV}_{-\alpha}, \alpha>0$, then for $n \geq 2$, as $x \rightarrow \infty$ we have

$$
\mathbb{P}\left(S_{n}(\mathbf{c})>x, X_{n, n} \leq x-\widetilde{\mathbf{c}} x\right)=o\left(\bar{F}(x)^{2}\right)
$$


and

$$
\mathbb{P}\left(S_{(n)}(\mathbf{c})>\widetilde{\mathbf{c}} x, X_{n, n}>x-\widetilde{\mathbf{c}} x\right)=\left(1+c_{2}\right)^{2 \alpha}\left(\begin{array}{l}
n \\
2
\end{array}\right) \bar{F}(x)^{2}(1+o(1)) .
$$

Define

$$
V_{\alpha}(x)=\int_{0}^{\widetilde{\boldsymbol{c}} x}\left(\left(1-\frac{u}{x}\right)^{-\alpha}-1\right) d F_{n}(u), \quad \mu_{F}(x)= \begin{cases}\overline{F_{n}}(x), & 0<\alpha<1, \\ x^{-1} \int_{0}^{x} u d F_{n}(u), & \alpha \geq 1 .\end{cases}
$$

for $x>0$. The following result extends Lemma 2.4 in Mao and Hu [22].

Lemma 2.5. If $\bar{F} \in \mathrm{RV}_{-\alpha}, \alpha>0$, then

$$
\lim _{x \rightarrow \infty} \frac{V_{\alpha}(x)}{\mu_{F}(x)}=h_{\alpha}:= \begin{cases}\widetilde{\mathbf{c}}^{-\alpha}\left(1-(1-\widetilde{\mathbf{c}})^{-\alpha}\right)+\alpha \int_{0}^{\widetilde{\mathbf{c}}} u^{-\alpha}(1-u)^{-(\alpha+1)} d u, & 0<\alpha<1, \\ \alpha, & \alpha \geq 1 .\end{cases}
$$

Furthermore, $\mu_{F} \in \mathrm{RV}_{-\alpha^{*}}$ with

$$
\mu_{F}(x) \sim \begin{cases}(n-1) c_{2}^{\alpha} \bar{F}(x), & 0<\alpha<1, \\ x^{-1} \mathbb{E}\left\{S_{n-1}(\mathbf{c})\right\}, & \alpha \geq 1, \mathbb{E}\{X\}<\infty, \\ (n-1) c_{2} x^{-1} \int_{0}^{x} u d F(u), & \alpha=1, \mathbb{E}\{X\}=\infty .\end{cases}
$$

as $x \rightarrow \infty$, where $\alpha^{*}=\min (1, \alpha)$.

\section{Main Results}

For $S_{n-1}(\mathbf{c})$ given by (2.2), denote $l=\lceil\alpha\rceil-1$ with $\lceil\alpha\rceil$ defined as before, i.e., the smallest integer which is greater than $\alpha$, and set

$$
\begin{aligned}
& d_{l+1}(x)=\sum_{j=0}^{l} \frac{(-1)^{j} \bar{F}^{(j)}(x)}{j !} \frac{\mathbb{E}\left\{S_{n-1}^{j}(\mathbf{c})\right\}}{\bar{F}(x)}, \quad R(x)= \begin{cases}\bar{F}(x), & \alpha \neq l+1, \\
x^{-\alpha} \int_{0}^{\widetilde{\mathbf{c}} x} u^{\alpha} d F_{n}(u), & \alpha=l+1,\end{cases} \\
& \kappa_{\mathbf{c}}= \begin{cases}\left(1+c_{2}\right)^{\alpha}\left(\left(1+c_{2}\right)^{\alpha}+2 \sum_{j=0}^{\infty} \frac{\Gamma(\alpha+j)}{\Gamma(\alpha) \Gamma(j+1)} \frac{\alpha \widetilde{\mathbf{c}}^{j}}{j-\alpha}\right), & \alpha \neq l+1, \\
\frac{2}{n-1} \frac{\Gamma(2 \alpha)}{\Gamma(\alpha) \Gamma(\alpha+1)}, & \alpha=l+1,\end{cases}
\end{aligned}
$$

with $\Gamma(\cdot)$ the Euler Gamma function. Under a smoothness varying and a second-order condition on $\bar{F}$, we establish the following higher-order and the second-order tail asymptotics of $S_{n}(\mathbf{c})$ in Theorem 3.1 and Theorem 3.5, respectively.

Theorem 3.1. If $\bar{F} \in \mathrm{SR}_{-\alpha,\lceil\alpha\rceil}, \alpha>0$, then

$$
\mathbb{P}\left(S_{n}(\mathbf{c})>x\right)=n \bar{F}(x)\left(d_{l+1}(x)+\frac{n-1}{2} \kappa_{\mathbf{c}} R(x)(1+o(1))\right)
$$

as $x \rightarrow \infty$, where $d_{l+1}, R$ and $\kappa_{\mathbf{c}}$ are given by (3.1) and (3.2), respectively.

Noting that $\bar{F} \in \mathrm{SR}_{-\alpha,\lceil\alpha\rceil}$ implies

$$
\mathbb{P}\left(X_{n, n}>x\right)=n \bar{F}(x)-\left(\begin{array}{l}
n \\
2
\end{array}\right) \bar{F}(x)^{2}(1+o(1)), \quad x \rightarrow \infty .
$$

Thus, combining with Theorem 3.1 we can derive the asymptotic expansion of $\Delta(x)=\mathbb{P}\left(S_{n}(\mathbf{c})>x\right)$ $\mathbb{P}\left(X_{n, n}>x\right)$ as follows. 
Corollary 3.2. Under the conditions of Theorem 3.1, we have

$$
\Delta(x)=n \bar{F}(x)\left(d_{l+1}(x)-1+\frac{n-1}{2} \tilde{\kappa}_{\mathbf{c}} R(x)(1+o(1))\right), \quad x \rightarrow \infty,
$$

where $\tilde{\kappa}_{\mathbf{c}}=\kappa_{\mathbf{c}}-\mathbb{I}\{\alpha \neq l+1\}$, and $d_{l+1}, R$ and $\kappa_{\mathbf{c}}$ are given by (3.1) and (3.2), respectively.

Remark 3.3. a) For general $c_{1}>0$, it is clear that $\mathbb{P}\left(S_{n}(\mathbf{c})>x\right)$ and $\Delta(x)$ can be asymptotically expanded as above, which are obtained by replacing $x$ and $\left(c_{2}, \ldots, c_{n}\right)$ by $x / c_{1}$ and $\left(c_{2} / c_{1}, \ldots, c_{n} / c_{1}\right)$ in the right-hand side of the above expansions.

b) For $\mathbf{c}=1$, Theorem 3.1 is in agreement with Theorem 3.5 in Albrecher et al. [1].

Most common distributions satisfy the smoothness varying condition in Theorem 3.1, e.g., Burr, Pareto, absolute student $t$, etc (see Examples in Section 5 ). In the literature, $\mathbb{E}\left\{S_{n-1}(\mathbf{c})\right\}$ is so-called the net premium, see Kremer [18. In our simulation study, we use empirical estimators to replace $\mathbb{E}\left\{S_{n-1}(\mathbf{c})\right\}$.

Theorem 3.1 is based on the fact that $\bar{F}$ has $l+1$ continuous derivatives. More generally, if we can find some asymptotic equivalent df $H$ such that $\bar{H}$ satisfies the smoothness varying condition and $\bar{H}$ is close enough to $\bar{F}$, then a similar result is derived as follows.

Corollary 3.4. If there exists a df $H$ such that $\bar{H} \in \mathrm{SR}_{-\alpha,\lceil\alpha\rceil}$, and $F-H$ is eventually with constant sign and $|F-H| \in \mathrm{RV}_{-(\alpha-\rho)}$ for some $\rho<0$, then for large $x$ we have

$$
\mathbb{P}\left(S_{n}(\mathbf{c})>x\right)=n \bar{F}(x)+n \bar{H}(x)\left(\tilde{d}_{l+1}(x)-1+\frac{n-1}{2} \kappa_{\mathbf{c}} R(x)(1+o(1))\right)
$$

and

$$
\Delta(x)=n \bar{H}(x)\left(\tilde{d}_{l+1}(x)-1+\frac{n-1}{2} \tilde{\kappa}_{\mathbf{c}} R(x)(1+o(1))\right),
$$

where $R$ and $\kappa_{\mathbf{c}}$ are given by (3.1) and (3.2), respectively, and

$$
\tilde{\kappa}_{\mathbf{c}}=\kappa_{c}-\mathbb{I}\{\alpha \neq l+1\}, \quad \tilde{d}_{l+1}(x)=\sum_{j=0}^{l} \frac{(-1)^{j} \bar{H}^{(j)}(x)}{j !} \frac{\mathbb{E}\left\{S_{n-1}^{j}(\mathbf{c})\right\}}{\bar{H}(x)} .
$$

To end this section, we establish the second-order tail asymptotics of $S_{n}(\mathbf{c})$ under the second-order regular variation condition on $\bar{F}$. For simplicity, set

$$
\phi_{\alpha}=2 \alpha c_{2}^{\alpha} \int_{0}^{\widetilde{\mathbf{c}}} u^{-\alpha}(1-u)^{-(\alpha+1)} d u-\left(1+c_{2}\right)^{2 \alpha}, \quad \alpha^{*}=\min (1, \alpha) .
$$

Theorem 3.5. If $\bar{F} \in 2 \mathrm{RV}_{-\alpha, \rho}, \alpha>0, \rho \leq 0$ with auxiliary function $A$, then for large $x$ we have

$$
\mathbb{P}\left(S_{n}(\mathbf{c})>x\right)=n \bar{F}(x)(1+\mathcal{E}(x)(1+o(1)))
$$

with

$$
\mathcal{E}(x)=\left(\frac{\left(1+c_{2}\right)^{\alpha}}{2}-1\right) \overline{F_{n}}(\widetilde{\mathbf{c}} x)+h_{\alpha} \mu_{F}(x)+o(A(x)),
$$

where $\mu_{F}$ and $h_{\alpha}$ are given by (2.6) and (2.7), and thus $|\mathcal{E}| \in \mathrm{RV}_{-\alpha^{*}}$.

Corollary 3.6. Under the conditions of Theorem 3.5, we have $\bar{F}_{S_{n}(\mathbf{c})} \in 2 \mathrm{RV}{ }_{-\alpha, \rho^{*}}$ with $\rho^{*}=\max (-1,-\alpha, \rho)$ and auxiliary function $A^{*}$ satisfying

$$
A^{*}(x)=A(x)+\alpha\left(1-\frac{\left(1+c_{2}\right)^{\alpha}}{2}\right) \overline{F_{n}}(\widetilde{\mathbf{c}} x)-\alpha^{*} h_{\alpha} \mu_{F}(x)
$$

with

$$
A^{*}(x) \sim \begin{cases}-\frac{n-1}{2} \alpha \phi_{\alpha} \bar{F}(x)+A(x) \mathbb{I}\{\rho=-\alpha\}, & \rho \leq-\alpha, 0<\alpha<1, \\ -\alpha \mu_{F}(x)+A(x) \mathbb{I}\{\rho=-1\}, & \rho \leq-1, \alpha \geq 1, \\ A(x), & \rho>-\alpha^{*}=-\min (1, \alpha),\end{cases}
$$

where $\mu_{F}, h_{\alpha}$ and $\phi_{\alpha}$ are given by (2.7) and (3.4), respectively. 
Remark 3.7. a) For $\alpha \in(0,1)$ and $c_{1}=c_{2}=1$, note that (see Albrecher et al. [1])

$$
\sum_{j=0}^{\infty} \frac{\Gamma(\alpha+j)}{2^{j} \Gamma(\alpha) \Gamma(j+1)} \frac{\alpha}{j-\alpha}=-2^{-\alpha-1}(1-2 \alpha) B(1-\alpha, 1-\alpha)-2^{\alpha-1}
$$

with $B(a, b):=\Gamma(a) \Gamma(b) / \Gamma(a+b)$ for some $a, b>0$. Further, as in Geluk et al. [14]

$$
2 \alpha \int_{0}^{1 / 2} u^{-\alpha}(1-u)^{-(\alpha+1)} d u=2^{2 \alpha}-\frac{\Gamma(1-\alpha)^{2}}{\Gamma(1-2 \alpha)}=2^{2 \alpha}-(1-2 \alpha) B(1-\alpha, 1-\alpha) .
$$

Consequently, Theorem 3.1] coincides with Theorem [3.5, i.e.,

$$
\mathbb{P}\left(S_{n}(\mathbf{c})>x\right)=n \bar{F}(x)\left(1-\frac{n-1}{2}(1-2 \alpha) B(1-\alpha, 1-\alpha) \bar{F}(x)(1+o(1))\right) .
$$

If $1-2 \alpha=0$, i.e., $\alpha=1 / 2$, then both Theorem 3.1 and Theorem 3.5 do not give the next term in the asymptotic expansion.

b) Theorem 3.5 and Corollary [3.6 include Theorem 3.4 and Theorem 3.5 in Mao and Hu [22], which consider only the case $\mathbf{c}=\mathbf{1}$ and $\rho \neq-\min (1, \alpha)$.

Theorem 3.5 and Corollary 3.6 may also be extended to the general case of $c_{1}>0$, see Remark 3.3 above. Additionally, we can conclude that the convergence rate of $\mathbb{P}\left(S_{n}(\mathbf{c})>x\right)-n \bar{F}(x)$ depends on $-\alpha^{*}$ and $\rho$. If $\rho=0$, the convergence rate can be arbitrarily slow, see Example 5.3 in Section 5

Remark 3.8. Let $X \sim F(x)=1-x^{-\alpha}, x>1$ with $\alpha>0$, i.e. the standard Pareto distribution. With cumbersome calculations, one can obtain that

$$
\begin{aligned}
\mathbb{P}\left(S_{2}(\mathbf{c})>x\right)= & 2 \bar{F}(x)\left[1+\left(\frac{\left(1+c_{2}\right)^{\alpha}}{2}-1\right) \bar{F}_{2}(\widetilde{\mathbf{c}} x)+\left(\left(1-\frac{c_{2}}{x}\right)^{-\alpha}-1\right)\right. \\
& \left.+\left(\widetilde{\mathbf{c}}^{-\alpha}\left(1-(1-\widetilde{\mathbf{c}})^{-\alpha}\right)+\alpha \int_{c_{2} / x}^{\widetilde{\mathbf{c}}} u^{-\alpha}(1-u)^{-(\alpha+1)} d u\right) \bar{F}_{2}(x)\right] \\
= & 2 \bar{F}(x)\left[1+\varepsilon^{*}(x)\right] .
\end{aligned}
$$

Then $\varepsilon^{*}(x) \sim \mathcal{E}(x)$. In particular, if $c_{1}=c_{2}=1$ and $\alpha=1$, then $\mathcal{E}(x)=(\ln x) / x$ and $\varepsilon^{*}(x)=(\ln (x-1)) / x$, which is in agreement with Ramsay [25].

\section{Applications}

Two applications of our main results are established in this section. The first one is to derive the second-order approximations of the ratio of two kinds of risk measures related to $S_{n}(\mathbf{c})$, and the second one is to establish the evaluation of the premium with respect to stop-loss and excess return on capital (ROC), respectively.

\subsection{Ratios of two kinds of risk measures}

In most application fields such as insurance and finance, Value-at-Risk (VaR) and conditional tail expectation (CTE) are two common risk measures, which are extensively studied, see Hua and Joe [16, Mao et al. 23] and the references therein. Tail Value-at-Risk (TVaR) and tail conditional tail expectation (TCTE) may be alternatives to measure risk, see Denuit et al. [11].

For the total risk $S_{n}(\mathbf{c})$ of $n$ independent portfolios $X_{i}$ 's with common df $F$, define

$$
C_{\mathrm{VaR}}(p)=\frac{\operatorname{VaR}_{p}\left(S_{n}(\mathbf{c})\right)}{\sum_{i=1}^{n} \operatorname{VaR}_{p}\left(X_{i}\right)}, \quad C_{\mathrm{CTE}}(p)=\frac{\operatorname{CTE}_{p}\left(S_{n}(\mathbf{c})\right)}{\sum_{i=1}^{n} \operatorname{CTE}_{p}\left(X_{i}\right)}, \quad p \in(0,1)
$$

where

$$
\operatorname{VaR}_{p}(X)=F^{\leftarrow}(p)=\inf \{x: F(x) \geq p\}, \quad \operatorname{CTE}_{p}(X)=\mathbb{E}\left\{X \mid X>\operatorname{VaR}_{p}(X)\right\}
$$


and $F^{\leftarrow}$ stands for the generalized inverse of $F$. For $\mathbf{c}=\mathbf{1}$, the quantities $C_{\mathrm{VaR}}(p)$ and $C_{\mathrm{CTE}}(p)$ are respectively called the risk concentrations based on the risk measures VaR and CTE at probability level $p$, and $1-C_{\operatorname{VaR}}(p)$ and $1-C_{\mathrm{CTE}}(p)$ are called the diversification benefits at probability level $p$. For more details, we refer to Degen et al. [10, Mao and $\mathrm{Hu}$ [22, Mao et al. 23] and the references therein.

Now, we consider the second-order expansions of the following ratios

$$
R_{\varphi}(p)=\frac{\mathbb{E}\left\{\varphi_{\kappa}\left(S_{n}(\mathbf{c})\right) \mid \kappa>p\right\}}{\varphi_{p}\left(S_{n}(\mathbf{c})\right)}=\frac{\int_{p}^{1} \varphi_{q}\left(S_{n}(\mathbf{c})\right) d q}{(1-p) \varphi_{p}\left(S_{n}(\mathbf{c})\right)}, \quad p \uparrow 1
$$

with risk measures $\varphi \in\{\mathrm{VaR}, \mathrm{CTE}\}$, where $\kappa \sim U(0,1)$. So, $R_{p}$ is just TVaR/VaR or TCTE/CTE.

Noting that

$$
R_{\mathrm{VaR}}(p)=\frac{\int_{p}^{1} C_{\mathrm{VaR}}(q) \frac{\operatorname{VaR}_{q}(X)}{\left.\operatorname{VaR}_{p}(X)\right)} d q}{(1-p) C_{\mathrm{VaR}}(p)}
$$

and

$$
R_{\mathrm{CTE}}(p)=\frac{\int_{p}^{1} C_{\mathrm{CTE}}(q) \frac{\mathrm{CTE}_{q}(X)}{\operatorname{CTE}_{p}(X)} d q}{(1-p) C_{\mathrm{CTE}}(p)},
$$

we shall first investigate the approximations of $C_{\mathrm{VaR}}(p)$ and $C_{\mathrm{CTE}}(p)$ in Theorem 4.1 below and then establish the second order approximation of the above two risk ratios in Theorem 4.3 .

Clearly, for some survival function $\bar{F} \in R V_{-\alpha}, \alpha>0$, we have (cf. Asimit et al. [5])

$$
\mathbb{P}\left(S_{n}(\mathbf{c})>x\right) \sim P\left(X_{n, n}>x\right) \sim n \bar{F}(x), \quad \text { as } x \rightarrow \infty
$$

implying

$$
C_{\mathrm{VaR}}(p) \sim C_{\mathrm{CTE}}(p) \sim n^{1 / \alpha-1}, \quad \text { as } p \uparrow 1 .
$$

As pointed out by Degen et al. 10] for $\mathbf{c}=\mathbf{1}$, the diversification benefits $C_{\mathrm{VaR}}(p)$ and $\left.C_{\mathrm{CTE}}(p)\right)$ may be very sensitive to $p$, i.e. small changes of $p$ may lead to large changes of $C_{\mathrm{VaR}}(p)$ and $C_{\mathrm{CTE}}(p)$ ), which motivates us to consider the convergence rate of $C_{\mathrm{VaR}}(p)-n^{1 / \alpha-1}$ and $C_{\mathrm{CTE}}(p)-n^{1 / \alpha-1}$, i.e., the second-order expansions of the risk concentrations of $S_{n}(\mathbf{c})$ based on the risk measures VaR and CTE. We will interpret $\left(n^{\rho / \alpha}-1\right) /(\rho / \alpha)$ as $\ln n$ for $\rho=0$, and keep the notation of $\mu_{F}$ and $\phi_{\alpha}$ given by (2.7) and (3.4), respectively.

Theorem 4.1. If $\bar{F} \in 2 \mathrm{RV}_{-\alpha, \rho}, \alpha>0, \rho \leq 0$ with auxiliary function $A$, then as $p \uparrow 1$

$$
C_{\mathrm{VaR}}(p)=n^{1 / \alpha-1}(1+\mathcal{E}(p)(1+o(1)))
$$

and further if $\alpha>1$, then

$$
C_{\mathrm{CTE}}(p)=n^{1 / \alpha-1}\left(1+\frac{\alpha-1}{\alpha-1-\max (-1, \rho)} \mathcal{E}(p)(1+o(1))\right),
$$

where

$$
\mathcal{E}(p)= \begin{cases}\frac{\left(1-n^{-1}\right) \phi_{\alpha}}{2 \alpha}(1-p)+\frac{1-n^{-1}}{\alpha^{2}} A\left(F^{\leftarrow}(p)\right) \mathbb{I}\{\rho=-\alpha\}, & \rho \leq-\alpha, 0<\alpha<1, \\ \frac{\mu_{F}\left(F^{\leftarrow}(p)\right)}{n^{1 / \alpha}}+\frac{1-n^{-1 / \alpha}}{\alpha} A\left(F^{\leftarrow}(p)\right) \mathbb{I}\{\rho=-1\}, & \rho \leq-1, \alpha \geq 1, \\ \frac{n^{\rho / \alpha}-1}{\alpha \rho} A\left(F^{\leftarrow}(p)\right), & \rho>-\min (1, \alpha) .\end{cases}
$$

Remark 4.2. a) Theorem 4.1 includes the bounded cases $\rho=-\min (1, \alpha)$ and $\mathbf{c}=\mathbf{1}$, generalizing Theorem 4.2 and Theorem 4.5 in $M a O$ and $H u$ [22].

b) For general $c_{1}>0$ we have

$$
C_{\mathrm{VaR}}(p)=c_{1} n^{1 / \alpha-1}(1+\mathcal{E}(p)(1+o(1))), \quad C_{\mathrm{CTE}}(p)=c_{1} n^{1 / \alpha-1}\left(1+\frac{(\alpha-1) \mathcal{E}(p)(1+o(1))}{\alpha-1-\max (-1, \rho)}\right),
$$

where $\mathcal{E}(p)$ is given by (4.2) with $\left(c_{2}, \ldots, c_{n}\right)$ is replaced by $\left(c_{2} / c_{1}, \ldots, c_{n} / c_{1}\right)$. 
Theorem 4.3. If $\bar{F} \in 2 \mathrm{RV}_{-\alpha, \rho}, \alpha>1, \rho \leq 0$ with auxiliary function $A$, then as $p \uparrow 1$

$$
R_{\mathrm{VaR}}(p)=\frac{\alpha}{\alpha-1}\left(1+\left(\frac{A\left(F^{\leftarrow}(p)\right)}{\alpha(\alpha-1-\rho)}+\frac{\max (\rho,-1)}{\alpha-1-\max (\rho,-1)} \mathcal{E}(p)\right)(1+o(1))\right)
$$

and

$$
R_{\mathrm{CTE}}(p)=\frac{\alpha}{\alpha-1}+\left(\frac{1}{(\alpha-1-\rho)^{2}} A\left(F^{\leftarrow}(p)\right)+\frac{\alpha \max (\rho,-1)}{(\alpha-1-\max (\rho,-1))^{2}} \mathcal{E}(p)\right)(1+o(1)),
$$

where $\mathcal{E}(p)$ is the one defined by (4.2) with $\alpha>1$.

\subsection{Evaluation of Premium under Stop-Loss and ROC rules}

In reinsurance applications, the evaluation of the premiums is of some interest. If we denote by $d$ the retention level, then the stop-loss premium of the reinsurance sums (1.1) is defined by $\mathbb{E}\left\{\max \left(S_{n}(\mathbf{c})-d, 0\right)\right\}$. Under the conditions of Theorem 3.5 with the additional restriction that $\alpha>1$, the asymptotic results given by Theorem 3.5 and Theorem 4.1 imply that $\mathbb{E}\left\{\max \left(S_{n}(\mathbf{c})-d, 0\right)\right\}$ satisfies

$$
\begin{aligned}
\mathbb{E}\left\{\max \left(S_{n}(\mathbf{c})-d, 0\right)\right\} & =\mathbb{P}\left(S_{n}(\mathbf{c})>d\right) \mathbb{E}\left\{S_{n}(\mathbf{c})-d \mid S_{n}(\mathbf{c})>d\right\} \\
& =n \bar{F}(d)(1+\mathcal{E}(d)(1+o(1))) \frac{d}{\alpha-1}\left(1+\frac{A^{*}(d)}{\alpha-1-\rho^{*}}(1+o(1))\right) \\
& =\frac{n d}{\alpha-1} \bar{F}(d)\left(1+\left(\mathcal{E}(d)+\frac{A^{*}(d)}{\alpha-1-\rho^{*}}\right)(1+o(1))\right), \quad d \rightarrow \infty,
\end{aligned}
$$

where $\mathcal{E}$ and $A^{*}$ are given by (3.5) and (3.6), respectively. In reality, the retention $d$ is usually taken as $\operatorname{VaR}_{p}\left(S_{n}(\mathbf{c})\right)$ with probability level $p$ close to 1 .

One may also evaluate the reinsurance premium, when the reinsurer fixes a performance measure such as, excess return on capital (ROC):

$$
\mathrm{ROC}=\frac{\text { Expected Profit }}{\text { Risk Capital }}=\frac{P-\mathbb{E}\left\{\varphi_{\kappa}\left(S_{n}(\mathbf{c})\right) \mid \kappa>p\right\}}{\varphi_{p}\left(S_{n}(\mathbf{c})\right)-\mathbb{E}\left\{\varphi_{\kappa}\left(S_{n}(\mathbf{c})\right) \mid \kappa>p\right\}},
$$

where $\kappa$ is uniformly distributed in $(0,1)$ and $P$ is so-called the reinsurance premium for a given risk measure $\varphi \in\{\mathrm{VaR}, \mathrm{CTE}\}$ at probability level $p \in(0,1)$. Thus, if $\varphi=\mathrm{VaR}, \mathrm{CTE}$ and $\mathrm{ROC}=\tau$, then the premiums $P \in\left\{P_{\mathrm{VaR}}(\tau), P_{\mathrm{CTE}}(\tau)\right\}$ hold by Theorem 4.1 and Theorem 4.3 as follows:

$$
\begin{aligned}
P_{\mathrm{VaR}}(\tau) & =n \operatorname{VaR}_{p}(X) C_{\mathrm{VaR}}(p)\left(\tau+(1-\tau) R_{\mathrm{VaR}}(p)\right) \\
& =n F^{\leftarrow}(p) C_{\mathrm{VaR}}(p)\left(\frac{\alpha-\tau}{\alpha-1}+\frac{\alpha(1-\tau)}{\alpha-1}\left(\frac{A\left(F^{\leftarrow}(p)\right)}{\alpha(\alpha-1-\rho)}+\frac{\max (\rho,-1)}{\alpha-1-\max (\rho,-1)} \mathcal{E}(p)\right)(1+o(1))\right)
\end{aligned}
$$

and

$$
\begin{aligned}
P_{\mathrm{CTE}}(\tau)= & n \operatorname{CTE}_{p}(X) C_{\mathrm{CTE}}(p)\left(\tau+(1-\tau) R_{\mathrm{CTE}}(p)\right) \\
= & n F^{\leftarrow}(p) C_{\mathrm{CTE}}(p)\left(1+\frac{A\left(F^{\leftarrow}(p)\right)}{\alpha(\alpha-1-\rho)}(1+o(1))\right) \\
& \times\left(\frac{\alpha-\tau}{\alpha-1}+(1-\tau)\left(\frac{A\left(F^{\leftarrow}(p)\right)}{(\alpha-1-\rho)^{2}}+\frac{\alpha \max (\rho,-1)}{(\alpha-1-\max (\rho,-1))^{2}} \mathcal{E}(p)\right)(1+o(1))\right)
\end{aligned}
$$

where $\mathcal{E}$ is given by (4.2) and the last step is due to Lemma 2.2 in Mao et al. 23.

In reality, we take $\varphi=\mathrm{VaR}, p=0.995$ under Solvency II and $\varphi=\mathrm{CTE}, p=0.99$ under Swiss Solvency Test. Meanwhile, sensible values $\tau$ for ROC are between $6 \%$ and $10 \%$. 


\section{Examples}

In this section, we first give several examples illustrating the second-order expansion of risk measures $C_{\mathrm{VaR}}, C_{\mathrm{CTE}}$ and the premiums $P_{\mathrm{VaR}}(\tau), P_{\mathrm{CTE}}(\tau)$ based on VaR, CTE and ROC, respectively.

Example 5.1. (Hall class) A df $F$ is said to belong to the Hall class if its survival function $\bar{F}$ has the following asymptotic representation

$$
\bar{F}(x)=k_{1} x^{-\alpha}\left(1+k_{2} x^{\rho}(1+o(1))\right), \quad x \rightarrow \infty,
$$

with $k_{1}>0, k_{2} \neq 0, \alpha>0$ and $\rho<0$. Such $F$ satisfies

a) $\bar{F} \in 2 \mathrm{RV}_{-\alpha, \rho}$ with auxiliary function $A(x) \sim k_{2} \rho x^{\rho}$ as $x \rightarrow \infty$;

b) $F^{\leftarrow}(p) \sim\left(\frac{1-p}{k_{1}}\right)^{-1 / \alpha}$ and $A\left(F^{\leftarrow}(p)\right) \sim k_{2} \rho\left(\frac{1-p}{k_{1}}\right)^{-\rho / \alpha}$ as $p \uparrow 1$.

Note that for $X$ with df $F$ we have $\mathbb{E}\{X\}<\infty$ for $\alpha>1$ and $\mathbb{E}\{X\}=\infty$ for $\alpha=1$. Hence by (2.8)

$$
\mu_{F}(x) \sim \begin{cases}x^{-1} \mathbb{E}\left\{S_{n-1}(\mathbf{c})\right\}, & \alpha>1, \\ k_{1}(n-1) c_{2} \frac{\ln x}{x}, & \alpha=1 .\end{cases}
$$

Consequently, Theorem 4.1. Remark 4.2 and equation (4.4) imply as $p \uparrow 1$

$$
\begin{aligned}
& C_{\mathrm{VaR}}(p)=c_{1} n^{1 / \alpha-1}(1+\mathcal{E}(p)(1+o(1))), \\
& P_{\mathrm{VaR}}(\tau)=n F^{\leftarrow}(p) C_{\mathrm{VaR}}(p)\left(\frac{\alpha-\tau}{\alpha-1}+\frac{\alpha(1-\tau)}{\alpha-1}\left(\frac{A\left(F^{\leftarrow}(p)\right)}{\alpha(\alpha-1-\rho)}+\frac{\max (\rho,-1)}{\alpha-1-\max (\rho,-1)} \mathcal{E}(p)\right)(1+o(1))\right)
\end{aligned}
$$

with $\tau \in(0,1)$ the ROC level, and $\phi_{\alpha}$ given by (3.4) with $c_{2}$ replaced by $c_{2} / c_{1}$ and

$$
\mathcal{E}(p)= \begin{cases}\frac{1-n^{-1}}{\alpha}\left(\frac{\phi_{\alpha}}{2}-\frac{k_{2}}{k_{1}} \mathbb{I}\{\rho=-\alpha\}\right)(1-p), & \rho \leq-\alpha, 0<\alpha<1, \\ \frac{c_{2}\left(n^{-1}-1\right)}{c_{1}}(1-p) \ln (1-p)(1+o(1)), & \rho \leq-1, \alpha=1, \\ \left(\frac{\mathbb{E}\left\{S_{n-1}(\mathbf{c})\right\}}{c_{1} n^{1 / \alpha}}+\frac{k_{2}\left(n^{-1 / \alpha}-1\right)}{\alpha} \mathbb{I}\{\rho=-1\}\right)\left(\frac{1-p}{k_{1}}\right)^{1 / \alpha}, & \rho \leq-1, \alpha>1, \\ \frac{k_{2}\left(n^{\rho / \alpha}-1\right)}{\alpha}\left(\frac{1-p}{k_{1}}\right)^{-\rho / \alpha}, & \rho>-\min (1, \alpha) .\end{cases}
$$

Similarly, for $\alpha>1$ we have

$$
\begin{aligned}
C_{\mathrm{CTE}}(p)= & c_{1} n^{1 / \alpha-1}\left(1+\frac{\alpha-1}{\alpha-1-\max (-1, \rho)} \mathcal{E}(p)(1+o(1))\right) \\
P_{\mathrm{CTE}}(\tau)= & n F^{\leftarrow}(p) C_{\mathrm{CTE}}(p)\left(1+\frac{A\left(F^{\leftarrow}(p)\right)}{\alpha(\alpha-1-\rho)}(1+o(1))\right) \\
& \times\left(\frac{\alpha-\tau}{\alpha-1}+(1-\tau)\left(\frac{A\left(F^{\leftarrow}(p)\right)}{(\alpha-1-\rho)^{2}}+\frac{\alpha \max (\rho,-1)}{(\alpha-1-\max (-1, \rho))^{2}} \mathcal{E}(p)\right)(1+o(1))\right) .
\end{aligned}
$$

Below is a short list of dfs that belong to Hall class:

a) $\operatorname{Burr}(a, b): \bar{F}(x)=\left(1+x^{b}\right)^{-a}, a, b>0$ with $\alpha=a b, \rho=-b$ and $k_{1}=1, k_{2}=-a$.

b) Hall/Weiss survival function: $\bar{F}(x)=x^{-\alpha}\left(1+x^{\rho}\right) / 2, \alpha>0, \rho<0$ and $k_{1}=1 / 2, k_{2}=1$.

c) Fréchet distribution function: $\bar{F}(x)=1-\exp \left(-x^{-\alpha}\right), \alpha>0$ with $\rho=-\alpha$ and $k_{1}=1, k_{2}=-1 / 2$.

d) $\operatorname{Pareto}(\alpha, \theta): \bar{F}(x)=\left(\frac{\theta}{x+\theta}\right)^{\alpha}, \alpha, \theta>0$ with $\rho=-1$ and $k_{1}=\theta^{\alpha}, k_{2}=-\alpha \theta$. 
Example 5.2. (Absolute student $t_{v}$ distribution) Let $X$ be a positive rv with probability density function $f$ given by

$$
f(x)=\frac{2 \Gamma((v+1) / 2)}{\sqrt{v \pi} \Gamma(v / 2)}\left(1+\frac{x^{2}}{v}\right)^{-(v+1) / 2}
$$

with $v>0$. In view of Proposition 6 in Hua and Joe [16]

$$
\bar{F}(x)=k_{1} x^{-v}\left(1+k_{2} x^{-2}(1+o(1))\right)
$$

where

$$
k_{1}=\frac{2 \Gamma((v+1) / 2)}{\sqrt{v \pi} \Gamma(v / 2)} v^{(v-1) / 2}, \quad k_{2}=-\frac{v^{2}(v+1)}{2(v+2)} .
$$

Consequently, $X$ has df $F$ that belongs to the Hall class with $\alpha=v, \rho=-2$ and $k_{1}, k_{2}$ as above. Hence one can use the formulas (5.2) and (5.3) to obtain the second-order risk measures based on VaR and CTE, respectively. Similar arguments hold for the second order approximations of the reinsurance premium in (4.4). On the other hand, a direct application of Theorem 4.1 and Remark 4.2 yields

$$
C_{\mathrm{VaR}}(p)=c_{1} n^{1 / \alpha-1}(1+\mathcal{E}(p)(1+o(1))), \quad p \uparrow 1
$$

with $\phi_{\alpha}$ given by (3.4) with $c_{2}$ replaced by $c_{2} / c_{1}$ and

$$
\mathcal{E}(p)= \begin{cases}\frac{\left(1-n^{-1}\right) \phi_{\alpha}}{2 \alpha}(1-p), & 0<\alpha<1, \\ \frac{c_{2}\left(1-n^{-1}\right)}{c_{1}} \frac{\int_{0}^{F^{\leftarrow}(p)} u d F(u)}{F^{\leftarrow}(p)}, & \alpha=1, \\ \frac{\mathbb{E}\left\{S_{n-1}(\mathbf{c})\right\}}{c_{1} n^{1 / \alpha}} \frac{1}{F^{\leftarrow}(p)}, & \alpha>1 .\end{cases}
$$

Further, for $\alpha>1$

$$
C_{\mathrm{CTE}}(p)=c_{1} n^{1 / \alpha-1}\left(1+\frac{\alpha-1}{\alpha} \mathcal{E}(p)(1+o(1))\right), \quad p \uparrow 1
$$

with $F^{\leftarrow}(p)=t_{v}^{\leftarrow}((p+1) / 2)$, where $t_{v}$ denotes the standard student $t$ distribution with $v$ degrees of freedom.

Example 5.3. (g-and-h distribution) A random variable $X$ possesses a g-and-h df if

$$
X=\kappa+\varsigma \frac{\mathrm{e}^{g Z}-1}{g} \exp \left(\frac{h Z^{2}}{2}\right), \quad(\kappa, g, h) \in \mathbb{R}^{3}, \varsigma>0,
$$

where $Z \sim N(0,1)$ with distribution function $\Phi$. Let $F$ denote the $\mathrm{df}$ of $X$ with $\kappa=0, \varsigma=1$ and $g>0$. In the light of Degen et al. [10] we have $\bar{F} \in 2 \mathrm{RV}_{-1 / h, 0}$ with auxiliary function $A(x)=h^{-2} a(1 / \bar{F}(x))$ and

$$
a\left(\frac{1}{1-p}\right)=\frac{g}{\Phi^{\leftarrow}(p)}(1+o(1)), \quad \text { as } p \uparrow 1 .
$$

By Theorem 4.1 the second-order asymptotics for two risk concentrations $C_{\mathrm{VaR}}(p)$ and $C_{\mathrm{CTE}}(p)$ are the same as follows

$$
C_{\mathrm{VaR}}(p)=C_{\mathrm{CTE}}(p)=c_{1} n^{h-1}\left(1+\frac{g \ln n}{\Phi^{\leftarrow}(p)}(1+o(1))\right), \quad \text { as } p \uparrow 1 .
$$

Further the second order approximations of $P_{\mathrm{VaR}}(\tau), P_{\mathrm{CTE}}(\tau)$ in (4.4) hold with $A\left(F^{\leftarrow}(p)\right)=g /\left(h^{2} \Phi^{\leftarrow}(p)\right)$. 


\section{Proofs}

Proof of Lemma 2.4 Let $c^{*}=\max \left(1, c_{2}, \ldots, c_{n}\right)$ and recall that $\widetilde{\mathbf{c}}=c_{2} /\left(1+c_{2}\right)$ and thus $c_{2}(1-\widetilde{\mathbf{c}})=\widetilde{\mathbf{c}}$. Then,

$$
\begin{aligned}
\mathbb{P}\left(S_{n}(\mathbf{c})>x, X_{n, n} \leq x-\widetilde{\mathbf{c}} x\right) & \leq n \mathbb{P}\left(S_{n}(\mathbf{c})>x, X_{n, n} \leq x-\widetilde{\mathbf{c}} x, X_{n, n}=X_{n}\right) \\
& \leq n \mathbb{P}\left(S_{n-1}(\mathbf{c})>\widetilde{\mathbf{c}} x, c_{2} X_{n-1, n-1} \leq \widetilde{\mathbf{c}} x, X_{n}>\frac{x}{n c^{*}}\right) \\
& =n \mathbb{P}\left(S_{n-1}(\mathbf{c})>\widetilde{\mathbf{c}} x, c_{2} X_{n-1, n-1} \leq \widetilde{\mathbf{c}} x\right) \mathbb{P}\left(X_{n}>\frac{x}{n c^{*}}\right) \\
& =o\left(\bar{F}(x)^{2}\right)
\end{aligned}
$$

as $x \rightarrow \infty$. The last step above is justified by the fact that $\mathbb{P}\left(S_{n-1}(\mathbf{c})>\widetilde{\mathbf{c}} x\right) \sim \mathbb{P}\left(c_{2} X_{n-1, n-1}>\widetilde{\mathbf{c}} x\right)$, which is shown in Asimit et al. [5].

Next,

$$
\begin{aligned}
& \mathbb{P}\left(S_{(n)}(\mathbf{c})>\widetilde{\mathbf{c}} x, X_{n, n}>x-\widetilde{\mathbf{c}} x\right) \\
= & \mathbb{P}\left(c_{2} X_{n-1, n}>\widetilde{\mathbf{c}} x, X_{n, n}>x-\widetilde{\mathbf{c}} x\right)+\mathbb{P}\left(X_{n, n}>x-\widetilde{\mathbf{c}} x, c_{2} X_{n-1, n} \leq \widetilde{\mathbf{c}} x, S_{(n)}(\mathbf{c})>\widetilde{\mathbf{c}} x\right) \\
= & \mathbb{P}\left(X_{n-1, n}>\widetilde{\mathbf{c}} x / c_{2}\right)+n \mathbb{P}\left(X_{n}>x-\widetilde{\mathbf{c}} x\right) \mathbb{P}\left(c_{2} X_{n-1, n-1} \leq \widetilde{\mathbf{c}} x, S_{n-1}(\mathbf{c})>\widetilde{\mathbf{c}} x\right) \\
= & \left(\begin{array}{l}
n \\
2
\end{array}\right) \bar{F}\left(\widetilde{\mathbf{c}} x / c_{2}\right)^{2}(1+o(1))=\left(1+c_{2}\right)^{2 \alpha}\left(\begin{array}{l}
n \\
2
\end{array}\right) \bar{F}(x)^{2}(1+o(1)),
\end{aligned}
$$

and thus the proof is complete.

Proof of Lemma 2.5. By partial integration and Potter bounds (cf. Proposition B.1.9 in de Haan and Ferreira [9]) for any $\alpha \in(0,1)$

$$
\begin{aligned}
\frac{V_{\alpha}(x)}{\overline{F_{n}}(x)} & =\left(1-(1-\widetilde{\mathbf{c}})^{-\alpha}\right) \frac{\overline{F_{n}}(\widetilde{\mathbf{c}} x)}{\overline{F_{n}}(x)}+\alpha \int_{0}^{\widetilde{\mathbf{c}}} \frac{\overline{F_{n}}(x u)}{\overline{F_{n}}(x)}(1-u)^{-(\alpha+1)} d u \\
& \rightarrow \widetilde{\mathbf{c}}^{-\alpha}\left(1-(1-\widetilde{\mathbf{c}})^{-\alpha}\right)+\alpha \int_{0}^{\widetilde{\mathbf{c}}} u^{-\alpha}(1-u)^{-\alpha-1} d u
\end{aligned}
$$

as $x \rightarrow \infty$. Next, for $\alpha \geq 1$ we borrow some argument from the proof of Lemma 2.4 in Mao and Hu [22]. Recall that $\overline{F_{n}}(x) \sim(n-1) c_{2}^{\alpha} \bar{F}(x)$ by Asimit et al. [5], $\overline{F_{n}} \in \mathrm{RV}_{-\alpha}$. Further since $\mu_{F} \in \mathrm{RV}_{-1}$ and by Karamata's theorem (cf. Resnick [26, p 17)

$$
\frac{x \overline{F_{n}}(x)}{\int_{0}^{x} \overline{F_{n}}(u) d u} \rightarrow 0, \quad \mu_{F}(x) \sim x^{-1} \int_{0}^{\widetilde{\mathbf{c}} x} \overline{F_{n}}(u) d u .
$$

Therefore, as $x \rightarrow \infty$

$$
\begin{aligned}
V_{\alpha}(x) & =\left(1-(1-\widetilde{\mathbf{c}})^{-\alpha}\right) \overline{F_{n}}(\widetilde{\mathbf{c}} x)+\frac{\alpha}{x} \int_{0}^{\widetilde{\mathbf{c}} x} \overline{F_{n}}(u)(1-u / x)^{-(\alpha+1)} d u \\
& =\frac{\alpha}{x} \int_{0}^{\widetilde{\mathbf{c}} x} \overline{F_{n}}(u)(1-u / x)^{-(\alpha+1)} d u(1+o(1)) .
\end{aligned}
$$

Since for $u \in(0, \widetilde{\mathbf{c}} x)$

$$
1+\frac{(\alpha+1) u}{x} \leq\left(1-\frac{u}{x}\right)^{-(\alpha+1)} \leq 1+\frac{(\alpha+1)(1-\widetilde{\mathbf{c}})^{-(\alpha+2)} u}{x}
$$

we have further

$$
\begin{aligned}
\liminf _{x \rightarrow \infty} \frac{V_{\alpha}(x)}{\mu_{F}(x)} & =\alpha \liminf _{x \rightarrow \infty} \frac{\int_{0}^{\widetilde{\mathbf{c}} x} \overline{F_{n}}(u)(1-u / x)^{-(\alpha+1)} d u}{\int_{0}^{\widetilde{\mathbf{c}} x} \overline{F_{n}}(u) d u} \\
& \geq \alpha+\alpha(\alpha+1) \lim _{x \rightarrow \infty} \frac{\int_{0}^{\widetilde{\mathbf{c}} x} u \overline{F_{n}}(u) d u}{x \int_{0}^{\widetilde{\mathbf{c}} x} \overline{F_{n}}(u) d u}
\end{aligned}
$$




$$
\begin{aligned}
& =\alpha+\alpha(\alpha+1) \widetilde{\mathbf{c}} \lim _{t \rightarrow \infty} \frac{\int_{0}^{t} u \overline{F_{n}}(u) d u}{t \int_{0}^{t} \overline{F_{n}}(u) d u} \\
& =\alpha+\alpha(\alpha+1) \widetilde{\mathbf{c}} \lim _{t \rightarrow \infty} \frac{t \overline{F_{n}}(t)}{t \overline{F_{n}}(t)+\int_{0}^{t} \overline{F_{n}}(u) d u}=\alpha
\end{aligned}
$$

and

$$
\limsup _{x \rightarrow \infty} \frac{V_{\alpha}(x)}{\mu_{F}(x)} \leq \alpha+\alpha(\alpha+1)(1-\widetilde{\mathbf{c}})^{-(\alpha+2)} \widetilde{\mathbf{c}} \lim _{t \rightarrow \infty} \frac{\int_{0}^{t} u \overline{F_{n}}(u) d u}{t \int_{0}^{t} \overline{F_{n}}(u) d u}=\alpha
$$

So,

$$
\lim _{x \rightarrow \infty} \frac{V_{\alpha}(x)}{\mu_{F}(x)}=\alpha
$$

For (2.8), noting that

$$
\frac{x \bar{F}(x)}{\int_{0}^{x} \bar{F}_{n}(u) d u} \rightarrow 0, \quad \bar{F}(x) \sim(n-1) c_{2}^{\alpha} \bar{F}(x)
$$

as $x \rightarrow \infty$. Hence the claim follows.

Proof of Theorem 3.1. First, we decompose $\mathbb{P}\left(S_{n}(\mathbf{c})>x\right)$ as follows

$$
\begin{aligned}
\mathbb{P}\left(S_{n}(\mathbf{c})>x\right)= & \mathbb{P}\left(S_{n}(\mathbf{c})>x, X_{n, n} \leq x-\widetilde{\mathbf{c}} x\right)+\mathbb{P}\left(S_{(n)}(\mathbf{c})>\widetilde{\mathbf{c}} x, X_{n, n}>x-\widetilde{\mathbf{c}} x\right) \\
& +\mathbb{P}\left(S_{(n)}(\mathbf{c}) \leq \widetilde{\mathbf{c}} x, X_{n, n}>x-S_{(n)}(\mathbf{c})\right) \\
=: \quad & I+I I+I I I .
\end{aligned}
$$

By Lemma 2.4.

$$
I+I I=\left(1+c_{2}\right)^{2 \alpha}\left(\begin{array}{l}
n \\
2
\end{array}\right) \bar{F}(x)^{2}(1+o(1)) .
$$

Next, we consider only the third term. Since $\bar{F}$ is $(l+1)$ th differentiable, the application of Taylor's expansion of $\bar{F}(x-u)$ at $x$ yields

$$
\begin{aligned}
& I I I=\mathbb{P}\left(S_{n}(\mathbf{c})>x, S_{(n)}(\mathbf{c}) \leq \widetilde{\mathbf{c}} x\right)=n \int_{0}^{\widetilde{\mathbf{c}} x} \bar{F}(x-u) d F_{n}(u) \\
= & n \sum_{j=0}^{l} \frac{(-1)^{j} \bar{F}^{(j)}(x)}{j !} \int_{0}^{\widetilde{\mathbf{c}} x} u^{j} d F_{n}(u)+n \int_{0}^{\widetilde{\mathbf{c}} x} \frac{(-1)^{l+1} u^{l+1} \bar{F}^{(l+1)}\left(x-u \xi_{u}^{x}\right)}{(l+1) !} d F_{n}(u) \\
= & n\left(\sum_{j=0}^{l} \frac{(-1)^{j} \bar{F}^{(j)}(x)}{j !} \mathbb{E}\left\{S_{n-1}^{j}(\mathbf{c})\right\}-\sum_{j=0}^{l} \frac{(-1)^{j} \bar{F}^{(j)}(x)}{j !} \int_{\widetilde{\mathbf{c}} x}^{\infty} u^{j} d F_{n}(u)+\int_{0}^{\widetilde{\mathbf{c}} x} \frac{(-u)^{l+1} \bar{F}^{(l+1)}\left(x-u \xi_{u}^{x}\right)}{(l+1) !} d F_{n}(u)\right) \\
= & n\left(\sum_{j=0}^{l} \frac{(-1)^{j} \bar{F}^{(j)}(x)}{j !} \mathbb{E}\left\{S_{n-1}^{j}(\mathbf{c})\right\}-\sum_{j=0}^{l} \frac{(-1)^{j}(\widetilde{\mathbf{c}} x)^{j} \bar{F}^{(j)}(x)}{j !} \int_{1}^{\infty} u^{j} d F_{n}(\widetilde{\mathbf{c}} x u)\right. \\
& n\left[\frac{(-1)^{l+1}(\widetilde{\mathbf{c}} x)^{l+1}}{(l+1) !} \int_{0}^{1} u^{l+1} \bar{F}^{(l+1)}\left(x-\widetilde{\mathbf{c}} x u \xi_{u}^{x}\right) d F_{n}(\widetilde{\mathbf{c}} x u)\right) \\
=: & n\left[J_{1}-J_{2}+J_{3}\right]
\end{aligned}
$$

for some $\xi_{u}^{x} \in(0,1)$. Note that $J_{1}=d_{l+1}(x) \bar{F}(x)$ (recall (3.1)), hence it remains to consider $J_{2}$ and $J_{3}$.

For $J_{2}$, noting that $\overline{F_{n}} \in \mathrm{RV}_{-\alpha}$, and using Potter bounds and the dominated convergence theorem, for $j \leq l<\alpha$ we have

$$
\begin{aligned}
& \int_{1}^{\infty} u^{j} d F_{n}(\widetilde{\mathbf{c}} x u)=\overline{F_{n}}(\widetilde{\mathbf{c}} x)+\int_{1}^{\infty} j u^{j-1} \overline{F_{n}}(\widetilde{\mathbf{c}} x u) d u \\
= & \overline{F_{n}}(\widetilde{\mathbf{c}} x)\left(1+\frac{j \int_{1}^{\infty} u^{j-1} \overline{F_{n}}(\widetilde{\mathbf{c}} x u) d u}{\overline{F_{n}}(\widetilde{\mathbf{c}} x)}\right)=\overline{F_{n}}(\widetilde{\mathbf{c}} x)\left(1+\frac{j}{\alpha-j}(1+o(1))\right)
\end{aligned}
$$


and by Karamata's theorem, we have

$$
\begin{aligned}
J_{2} & =\sum_{j=0}^{l} \frac{(-1)^{j}(\widetilde{\mathbf{c}} x)^{j} \bar{F}^{(j)}(x)}{j !}\left(\overline{F_{n}}(\widetilde{\mathbf{c}} x) \frac{\alpha}{\alpha-j}(1+o(1))\right) \\
& =\bar{F}(x) \overline{F_{n}}(\widetilde{\mathbf{c}} x) \sum_{j=0}^{l} \frac{(-1)^{j} x^{j} \bar{F}^{(j)}(x)}{j ! \bar{F}(x)} \frac{\alpha \widetilde{\mathbf{c}}^{j}}{\alpha-j}(1+o(1)) \\
& =\bar{F}(x) \overline{F_{n}}(\widetilde{\mathbf{c}} x) \sum_{j=0}^{l} \frac{\Gamma(\alpha+j)}{\Gamma(\alpha) \Gamma(j+1)} \frac{\alpha \widetilde{\mathbf{c}}^{j}}{\alpha-j}(1+o(1)) .
\end{aligned}
$$

Next, we consider $J_{3}$ defined in (6.3). Recall the definition of $\xi_{u}^{x}$ in the remainder of the Taylor's expansion in (6.3), the integral of $J_{3}$ is

$$
\begin{aligned}
& \frac{\bar{F}(x-\widetilde{\mathbf{c}} x u)-\sum_{j=0}^{l} \frac{(\widetilde{\mathbf{c}} x u)^{j}}{(-1)^{j} j !} \bar{F}^{j}(x)}{(-1)^{l+1}(\widetilde{\mathbf{c}} x)^{l+1} \bar{F}^{(l+1)}(x)} \\
= & \widetilde{\mathbf{c}}^{-(l+1)}\left(\frac{(-1)^{l+1} \bar{F}(x)}{x^{l+1} \bar{F}^{(l+1)}(x)} \frac{\bar{F}(x-\widetilde{\mathbf{c}} x u)}{\bar{F}(x)}-\sum_{j=0}^{l} \frac{(-1)^{l+1-j} \bar{F}^{(j)}(x)}{j ! x^{l+1-j} \bar{F}^{(l+1)}(x)}(\widetilde{\mathbf{c}} u)^{j}\right) \\
\rightarrow & \frac{\Gamma(\alpha) \widetilde{\mathbf{c}}^{-(l+1)}}{\Gamma(\alpha+l+1)}\left((1-\widetilde{\mathbf{c}} u)^{-\alpha}-\sum_{j=0}^{l} \frac{\Gamma(\alpha+j)}{\Gamma(\alpha) \Gamma(j+1)}(\widetilde{\mathbf{c}} u)^{j}\right) \\
= & \frac{\Gamma(\alpha) \widetilde{\mathbf{c}}^{-(l+1)}}{\Gamma(\alpha+l+1)} \sum_{j=l+1}^{\infty} \frac{\Gamma(\alpha+j)}{\Gamma(\alpha) \Gamma(j+1)}(\widetilde{\mathbf{c}} u)^{j}
\end{aligned}
$$

holds uniformly for $u \in(0,1)$ as $x \rightarrow \infty$. First, we consider $\alpha \neq l+1$. To derive this, by the uniform convergence theorem for regularly varying functions

$$
\begin{aligned}
J_{3} & =\frac{(-1)^{l+1} x^{l+1} \bar{F}^{(l+1)}(x)}{\bar{F}(x)} \frac{\Gamma(\alpha) \bar{F}(x)}{\Gamma(\alpha+l+1)} \sum_{j=l+1}^{\infty} \frac{\Gamma(\alpha+j) \widetilde{\mathbf{c}}^{j}}{\Gamma(\alpha) \Gamma(j+1)} \int_{0}^{1} u^{j} d F_{n}(\widetilde{\mathbf{c}} x u)(1+o(1)) \\
& =\bar{F}(x) \overline{F_{n}}(\widetilde{\mathbf{c}} x)\left(\sum_{j=l+1}^{\infty} \frac{\Gamma(\alpha+j)}{\Gamma(\alpha) \Gamma(j+1)} \frac{\alpha \widetilde{\mathbf{c}}^{j}}{j-\alpha}\right)(1+o(1)) .
\end{aligned}
$$

The last step is due to

$$
\begin{aligned}
\int_{0}^{1} u^{j} d F_{n}(\widetilde{\mathbf{c}} x u) & =\overline{F_{n}}(\widetilde{\mathbf{c}} x)\left(\int_{0}^{1} \frac{j u^{j-1} \overline{F_{n}}(\widetilde{\mathbf{c}} x u)}{\overline{F_{n}}(\widetilde{\mathbf{c}} x)} d u-1\right) \\
& =\overline{F_{n}}(\widetilde{\mathbf{c}} x) \frac{\alpha}{j-\alpha}(1+o(1))
\end{aligned}
$$

which follows from Potter bounds for $j>\alpha$ and the dominated convergence theorem.

Now, we consider the case of $\alpha=l+1$. Noting that the left-hand side of (6.6) is dominated by

$$
\int_{0}^{1} u^{\alpha} d F_{n}(\widetilde{\mathbf{c}} x u)=\frac{\int_{0}^{\widetilde{\mathbf{c}} x} u^{\alpha} d F_{n}(u)}{(\widetilde{\mathbf{c}} x)^{\alpha}}
$$

for all $j>\alpha$. Consequently,

$$
J_{3}=\frac{\Gamma(2 \alpha) \bar{F}(x)}{\Gamma(\alpha) \Gamma(\alpha+1)} \frac{\int_{0}^{\widetilde{\mathbf{c}} x} u^{\alpha} d F_{n}(u)}{x^{\alpha}}(1+o(1)) .
$$


Combining (6.3), (6.4) and (6.5) for $\alpha \neq l+1$, we have

$$
I I I=n \bar{F}(x)\left(d_{l+1}+\sum_{j=0}^{\infty} \frac{\Gamma(\alpha+j)}{\Gamma(\alpha) \Gamma(j+1)} \frac{\alpha \widetilde{\mathbf{c}}^{j}}{j-\alpha} \overline{F_{n}}(\widetilde{\mathbf{c}} x)(1+o(1))\right) .
$$

For $\alpha=l+1$, by (6.3), (6.4) and (6.8) and using Karamata's theorem

$$
\begin{aligned}
I I I & =n \bar{F}(x)\left(d_{l+1}+\left(\sum_{j=0}^{l} \frac{\Gamma(\alpha+j)}{\Gamma(\alpha) \Gamma(j+1)} \frac{\alpha \widetilde{\mathbf{c}}^{j}}{j-\alpha} \overline{F_{n}}(\widetilde{\mathbf{c}} x)+\frac{\Gamma(2 \alpha)}{\Gamma(\alpha) \Gamma(\alpha+1)} \frac{\int_{0}^{\widetilde{\mathbf{c}} x} u^{\alpha} d F_{n}(u)}{x^{\alpha}}\right)(1+o(1))\right) \\
& =n \bar{F}(x)\left(d_{l+1}(x)+\frac{\Gamma(2 \alpha)}{\Gamma(\alpha) \Gamma(\alpha+1)} \frac{\int_{0}^{\widetilde{\mathbf{c}} x} u^{\alpha} d F_{n}(u)}{x^{\alpha}}(1+o(1))\right)
\end{aligned}
$$

and thus the proof is complete.

Proof of Corollary 3.4. Clearly, (6.2) holds for $\bar{H}$ due to $\bar{F} \in \mathrm{RV}_{-\alpha},|F-H| \in \mathrm{RV}_{-(\alpha-\rho)}$ with $\rho<0$ and Lemma 2.4 For the third term III in (6.1), we split it as follows

$$
I I I=n \int_{0}^{\widetilde{\mathbf{c}} x} \bar{H}(x-u) d F_{n}(u)+n \int_{0}^{\widetilde{\mathbf{c}} x}(\bar{F}(x-u)-\bar{H}(x-u)) d F_{n}(u)=: n\left(I I I_{1}+I I I_{2}\right) .
$$

For $I I I_{1}$, by Taylor's expansion for $\bar{H}$ at $x$, for $\alpha \neq l+1$ we have

$$
I I I_{1}=\bar{H}(x)\left(\tilde{d}_{l+1}(x)+\overline{F_{n}}(\widetilde{\mathbf{c}} x)\left(\sum_{j=0}^{\infty} \frac{\Gamma(\alpha+j)}{\Gamma(\alpha) \Gamma(j+1)} \frac{\alpha \widetilde{\mathbf{c}}^{j}}{j-\alpha}\right)(1+o(1))\right) .
$$

Further, for $\alpha=l+1$

$$
I I I_{1}=\bar{H}(x)\left(\tilde{d}_{l+1}(x)+\frac{\Gamma(2 \alpha)}{\Gamma(\alpha) \Gamma(\alpha+1)} \frac{\int_{0}^{\widetilde{\mathbf{c}} x} u^{\alpha} d F_{n}(u)}{x^{\alpha}}(1+o(1))\right),
$$

where $\tilde{d}_{l+1}$ defined by (3.3).

Since $H$ is eventually continuous and $\bar{H}-\bar{F}$ is eventually positive or negative, the uniform convergence theorem implies

$$
\begin{aligned}
& I I I_{2}=(\bar{F}(x)-\bar{H}(x)) \int_{0}^{\widetilde{\mathbf{c}} x}\left(1-\frac{u}{x}\right)^{-(\alpha-\rho)} d F_{n}(u)(1+o(1)) \\
& =(\bar{F}(x)-\bar{H}(x))\left(1-(1-\widetilde{\mathbf{c}})^{-(\alpha-\rho)} \overline{F_{n}}(\widetilde{\mathbf{c}} x)+\frac{\alpha-\rho}{x}\left(\int_{0}^{\widetilde{\mathbf{c}} x}\left(1-\frac{u}{x}\right)^{-(\alpha-\rho+1)} \overline{F_{n}}(u) d u\right)(1+o(1))\right) \\
& =(\bar{F}(x)-\bar{H}(x))\left(1-(1-\widetilde{\mathbf{c}})^{-(\alpha-\rho)} \overline{F_{n}}(\widetilde{\mathbf{c}} x)+(\alpha-\rho) \overline{F_{n}}(x)\left(\int_{0}^{\widetilde{\mathbf{c}}}(1-u)^{-(\alpha-\rho+1)} \frac{\overline{F_{n}}(u x)}{\overline{F_{n}}(x)} d u\right)(1+o(1))\right) \\
& =(\bar{F}(x)-\bar{H}(x))(1+o(1)) \\
& =o(\bar{H}(x))
\end{aligned}
$$

duo to $\bar{H} \in \mathrm{RV}_{-\alpha}$ and $|\bar{F}-\bar{H}| \in \mathrm{RV}_{-(\alpha-\rho)}$ with $\rho<0$. Therefore, the proof is complete.

Proof of Theorem 3.5. By the decomposition as in (6.1), it follows from Lemma 2.4 that

$$
I+I I=\left(1+c_{2}\right)^{2 \alpha}\left(\begin{array}{l}
n \\
2
\end{array}\right) \bar{F}(x)^{2}(1+o(1))=\frac{\left(1+c_{2}\right)^{\alpha}}{2} n \bar{F}(x) \overline{F_{n}}(\widetilde{\mathbf{c}} x)(1+o(1)) .
$$

Next rewrite $I I I$ as

$$
I I I=n \int_{0}^{\widetilde{\mathbf{c}} x} \bar{F}(x-u) d F_{n}(u)
$$




$$
\begin{aligned}
& =n \bar{F}(x)\left(1-\overline{F_{n}}(\widetilde{\mathbf{c}} x)+A(x) \int_{0}^{\widetilde{\mathbf{c}} x} \frac{\bar{F}(x-u) / \bar{F}(x)-(1-u / x)^{-\alpha}}{A(x)} d F_{n}(u)+\int_{0}^{\widetilde{\mathbf{c}} x}\left(\left(1-\frac{u}{x}\right)^{-\alpha}-1\right) d F_{n}(u)\right) \\
& =: n \bar{F}(x)\left(1-\overline{F_{n}}(\widetilde{\mathbf{c}} x)+A(x) \int_{0}^{\widetilde{\mathbf{c}} x} \psi_{x}\left(1-\frac{u}{x}\right) d F_{n}(u)+V_{\alpha}(x)\right)
\end{aligned}
$$

with

$$
\psi_{x}\left(1-\frac{u}{x}\right)=\frac{\bar{F}(x-u) / \bar{F}(x)-(1-u / x)^{-\alpha}}{A(x)}, \quad V_{\alpha}(x)=\int_{0}^{\widetilde{\mathbf{c}} x}\left(\left(1-\frac{u}{x}\right)^{-\alpha}-1\right) d F_{n}(u) .
$$

Since $\bar{F} \in 2 \mathrm{RV}_{-\alpha, \rho}$, it follows from Lemma 5.2 in Draisma et al. [12] that for all $\epsilon>0$, there exists $x_{0}=x_{0}(\epsilon)>0$ such that for all $x>x_{0}$ and $u \in(0, \widetilde{\mathbf{c}} x)$

$$
\left|\psi_{x}\left(1-\frac{u}{x}\right)-H_{-\alpha, \rho}\left(1-\frac{u}{x}\right)\right| \leq \epsilon\left(C_{1}+C_{2}(1-u / x)^{-\alpha}+C_{3}(1-u / x)^{-\alpha+\rho-\epsilon}\right),
$$

where $H_{-\alpha, \rho}$ is given by (2.1), and $C_{1}, C_{2}, C_{3}$ are three positive constants, independent with $x$ and $u$. Therefore, by the dominated convergence theorem

$$
\lim _{x \rightarrow \infty} \int_{0}^{\widetilde{\mathbf{c}} x} \psi_{x}\left(1-\frac{u}{x}\right) d F_{n}(u)=\int_{0}^{\infty} \lim _{x \rightarrow \infty} H_{-\alpha, \rho}\left(1-\frac{u}{x}\right) d F_{n}(u)=0 .
$$

Finally by Lemma 2.5 $V_{\alpha}(x)=h_{\alpha} \mu_{F}(x)(1+o(1))$, and thus the proof is complete.

Proof of Theorem 4.1] Let $U(t)=\inf \{y: F(y) \geq 1-1 / t\}$ and $\bar{G}(x)=\mathbb{P}\left(S_{n}(\mathbf{c})>x\right)$. Set $x_{p}=G^{\leftarrow}(p)$ for some given $p \in(0,1)$, i.e. the Value-at-Risk of $S_{n}(\mathbf{c})$ at probability level $p$, denoted by $\operatorname{VaR}_{p}\left(S_{n}(\mathbf{c})\right)$. Then $\bar{G}\left(x_{p}\right)=1-p$ and

$$
C_{\mathrm{VaR}}(p)=\frac{\operatorname{VaR}_{p}\left(S_{n}(\mathbf{c})\right)}{n \operatorname{VaR}_{p}(X)}=\frac{x_{p}}{n U(1 /(1-p))}=\frac{U\left(1 / \bar{F}\left(x_{p}\right)\right)}{n U\left(1 / \bar{G}\left(x_{p}\right)\right)} .
$$

Note that by Theorem 2.3.9 in de Haan and Ferreira 9$] U \in 2 \mathrm{RV}_{1 / \alpha, \rho / \alpha}$ with auxiliary function $\alpha^{-2} A(U)$. This together with Theorem 3.5 yields

$$
\lim _{p \rightarrow 1} \frac{\frac{U\left(1 / \bar{F}\left(x_{p}\right)\right)}{n U\left(1 / \bar{G}\left(x_{p}\right)\right)}-\frac{1}{n}\left(\frac{\bar{G}\left(x_{p}\right)}{\bar{F}\left(x_{p}\right)}\right)^{1 / \alpha}}{\alpha^{-2} A\left(U\left(1 / \bar{G}\left(x_{p}\right)\right)\right)}=n^{1 / \alpha-1} \frac{n^{\rho / \alpha}-1}{\rho / \alpha}, \quad \lim _{p \rightarrow 1} \frac{G^{\leftarrow}(p)}{F^{\leftarrow}(p)}=n^{1 / \alpha} .
$$

Hence with $\mathcal{E}$ given by Theorem 3.5 we have

$$
\begin{aligned}
C_{\mathrm{VaR}}(p) & =\frac{1}{n}\left(\frac{\bar{G}\left(x_{p}\right)}{\bar{F}\left(x_{p}\right)}\right)^{1 / \alpha}+n^{1 / \alpha-1} \frac{n^{\rho / \alpha}-1}{\alpha \rho} A(U(1 /(1-p)))(1+o(1)) \\
& =\frac{1}{n}\left(\frac{n \bar{F}\left(x_{p}\right)}{\bar{F}\left(x_{p}\right)}\left(1+\mathcal{E}\left(x_{p}\right)(1+o(1))\right)\right)^{1 / \alpha}+n^{1 / \alpha-1} \frac{n^{\rho / \alpha}-1}{\alpha \rho} A\left(F^{\leftarrow}(p)\right)(1+o(1)) \\
& =n^{1 / \alpha-1}\left(1+\frac{\mathcal{E}\left(x_{p}\right)}{\alpha}(1+o(1))+\frac{n^{\rho / \alpha}-1}{\alpha \rho} A\left(F^{\leftarrow}(p)\right)(1+o(1))\right) \\
& =n^{1 / \alpha-1}\left(1+\left(\frac{n^{-\alpha^{*} / \alpha}}{\alpha} \mathcal{E}\left(F^{\leftarrow}(p)\right)+\frac{n^{\rho / \alpha}-1}{\alpha \rho} A\left(F^{\leftarrow}(p)\right)\right)(1+o(1))\right), \quad p \rightarrow 1,
\end{aligned}
$$

where $\alpha^{*}=\min (1, \alpha)$. Since $|A| \in \mathrm{RV}_{\rho}$ and $|\mathcal{E}| \in \mathrm{RV}_{-\alpha^{*}}$, we consider the following three cases, i.e., a) $\rho \leq-\alpha, 0<\alpha<1 ; b) \rho \leq-1, \alpha \geq 1$ and $c) \rho>-\alpha^{*}$ in turn.

a) For $\rho \leq-\alpha$ and $0<\alpha<1$. It follows from $\rho \leq-\alpha^{*}=-\alpha$ and $\mathcal{E}(x) \sim \frac{(n-1) \phi_{\alpha}}{2} \bar{F}(x)$ that

$$
\begin{aligned}
C_{\mathrm{VaR}}(p) & =n^{1 / \alpha-1}\left(1+\left(\frac{n^{-1}}{\alpha} \frac{(n-1) \phi_{\alpha}}{2} \bar{F}\left(F^{\leftarrow}(p)\right)+\frac{n^{-\alpha / \alpha}-1}{\alpha(-\alpha)} A\left(F^{\leftarrow}(p)\right) \mathbb{I}\{\rho=-\alpha\}\right)(1+o(1))\right) \\
& =n^{1 / \alpha-1}\left(1+\left(\frac{\left(1-n^{-1}\right) \phi_{\alpha}}{2 \alpha}(1-p)+\frac{1-n^{-1}}{\alpha^{2}} A\left(F^{\leftarrow}(p)\right) \mathbb{I}\{\rho=-\alpha\}\right)(1+o(1))\right) .
\end{aligned}
$$


b) For $\rho \leq-1$ and $\alpha \geq 1$. It follows from $\rho \leq-\alpha^{*}=-1$ and $\mathcal{E}(x) \sim h_{\alpha} \mu_{F}(x)=\alpha \mu_{F}(x)$ that

$$
\begin{aligned}
C_{\mathrm{VaR}}(p) & =n^{1 / \alpha-1}\left(1+\left(\frac{n^{-1 / \alpha}}{\alpha} \alpha \mu_{F}\left(F^{\leftarrow}(p)\right)+\frac{n^{-1 / \alpha}-1}{\alpha(-1)} A\left(F^{\leftarrow}(p)\right) \mathbb{I}\{\rho=-1\}\right)(1+o(1))\right) \\
& =n^{1 / \alpha-1}\left(1+\left(\frac{\mu_{F}\left(F^{\leftarrow}(p)\right)}{n^{1 / \alpha}}+\frac{1-n^{-1 / \alpha}}{\alpha} A\left(F^{\leftarrow}(p)\right) \mathbb{I}\{\rho=-1\}\right)(1+o(1))\right) .
\end{aligned}
$$

c) Clearly, for $\rho>-\alpha^{*}$

$$
C_{\mathrm{VaR}}(p)=n^{1 / \alpha-1}\left(1+\frac{n^{\rho / \alpha}-1}{\alpha \rho} A\left(F^{\leftarrow}(p)\right)(1+o(1))\right)
$$

Thus, the claim of $C_{\mathrm{VaR}}(p)$ follows from $\left.\left.a\right), b\right)$ and $c$ ).

Next, we derive the asymptotics of $C_{\mathrm{CTE}}(p)$ as $p \rightarrow 1$. By Lemma 2.2 in Mao et al. 23.

$$
\operatorname{CTE}_{p}(X)=\frac{\alpha}{\alpha-1} \operatorname{VaR}_{p}(X)\left(1+\frac{1}{\alpha(\alpha-1-\rho)} A\left(\operatorname{VaR}_{p}(X)\right)(1+o(1))\right) .
$$

Further, Theorem 3.4 implies

$$
\operatorname{CTE}_{p}\left(S_{n}(\mathbf{c})\right)=\frac{\alpha}{\alpha-1} \operatorname{VaR}_{p}\left(S_{n}(\mathbf{c})\right)\left(1+\frac{1}{\alpha\left(\alpha-1-\rho^{*}\right)} A^{*}\left(\operatorname{VaR}_{p}\left(S_{n}(\mathbf{c})\right)\right)(1+o(1))\right),
$$

hence we have

$$
\begin{aligned}
C_{\mathrm{CTE}}(p) & =C_{\mathrm{VaR}}(p) \frac{1+\frac{1}{\alpha\left(\alpha-1-\rho^{*}\right)} A^{*}\left(\operatorname{VaR}_{p}\left(S_{n}(\mathbf{c})\right)\right)(1+o(1))}{1+\frac{1}{\alpha(\alpha-1-\rho)} A\left(\operatorname{VaR}_{p}(X)\right)(1+o(1))} \\
& =C_{\mathrm{VaR}}(p)\left(1+\left(\frac{n^{\rho^{*} / \alpha}}{\alpha\left(\alpha-1-\rho^{*}\right)} A^{*}\left(F^{\leftarrow}(p)\right)-\frac{1}{\alpha(\alpha-1-\rho)} A\left(F^{\leftarrow}(p)\right)\right)(1+o(1))\right) .
\end{aligned}
$$

The rest proof follows the similar arguments as for $C_{\mathrm{VaR}}(p)$.

Proof of Theorem 4.3 By Theorem 4.1 we have

$$
\begin{aligned}
R_{\mathrm{VaR}}(p) & =(1-\mathcal{E}(p)(1+o(1))) \frac{1}{1-p} \int_{p}^{1}(1+\mathcal{E}(q)(1+o(1))) \frac{U(1 /(1-q)}{U(1 /(1-p))} d q \\
& =(1-\mathcal{E}(p)(1+o(1))) \int_{1}^{\infty} t^{-2}\left(1+\mathcal{E}(p) t^{\max (\rho,-1) / \alpha}(1+o(1))\right) \frac{U(t /(1-p))}{U(1 /(1-p))} d t
\end{aligned}
$$

since $\mathcal{E}(q)$ is a regular varying function with index $\max (\rho,-1) / \alpha$ at 1 . Note that $U \in 2 R V_{1 / \alpha, \rho / \alpha}$ with auxiliary function $\alpha^{-2} A(U)$, we have

$$
\begin{aligned}
R_{\mathrm{VaR}}(p)= & (1-\mathcal{E}(p)(1+o(1))) \int_{1}^{\infty} t^{-2} \frac{U(t /(1-p))}{U(1 /(1-p))} d t+\left(\int_{1}^{\infty} t^{(1+\max (\rho,-1)) / \alpha-2} d t\right) \mathcal{E}(p)(1+o(1)) \\
= & (1-\mathcal{E}(p)(1+o(1)))\left(\int_{1}^{\infty} t^{1 / \alpha-2} d t+\alpha^{-2} A(U(1 /(1-p))) \int_{1}^{\infty} t^{1 / \alpha-2} \frac{t^{\rho / \alpha}-1}{\rho / \alpha} d t\right. \\
& \left.+\alpha^{-2} A(U(1 /(1-p))) \int_{1}^{\infty} t^{-2}\left(\frac{U(t /(1-p)) / U(1 /(1-p))-t^{1 / \alpha}}{\alpha^{-2} A(U(1 /(1-p)))}-t^{1 / \alpha} \frac{t^{\rho / \alpha}-1}{\rho / \alpha}\right) d t\right) \\
& +\frac{\alpha \mathcal{E}(p)}{\alpha-1-\max (\rho,-1)}(1+o(1)) \\
= & \frac{\alpha \mathcal{E}(p)}{\alpha-1-\max (\rho,-1)}(1+o(1))+(1-\mathcal{E}(p)(1+o(1)))\left(\frac{\alpha}{\alpha-1}+\frac{A\left(F^{\leftarrow}(p)\right)}{(\alpha-1)(\alpha-1-\rho)}(1+o(1))\right) \\
= & \frac{\alpha}{\alpha-1}\left(1+\frac{\max (\rho,-1)}{\alpha-1-\max (\rho,-1)} \mathcal{E}(p)(1+o(1))+\frac{A\left(F^{\leftarrow}(p)\right)}{\alpha(\alpha-1-\rho)}(1+o(1))\right), \quad p \uparrow 1
\end{aligned}
$$


where the last step follows by the dominated convergence theorem and the uniform inequality of Theorem 2.3 .9 by de Haan and Ferreira 9 .

For the second-order asymptotic of $R_{\mathrm{CTE}}(p)$, noting that

$$
\operatorname{CTE}_{p}(X)=\frac{\alpha}{\alpha-1}\left(1+\frac{1}{\alpha(\alpha-1-\rho)} A\left(F^{\leftarrow}(p)\right)(1+o(1))\right) \operatorname{VaR}_{p}(X), \quad \alpha>1
$$

due to Lemma 2.2 in Mao et al. 23. So, similar argument as for $R_{\mathrm{VaR}}(p)$ together with Theorem 4.1 yields that

$$
R_{\mathrm{CTE}}(p)=\frac{\alpha}{\alpha-1}+\left(\frac{1}{(\alpha-1-\rho)^{2}} A\left(F^{\leftarrow}(p)\right)+\frac{\alpha \max (\rho,-1)}{(\alpha-1-\max (\rho,-1))^{2}} \mathcal{E}(p)\right)(1+o(1))
$$

as $p \rightarrow 1$. The claimed result follows.

\section{References}

[1] H. Albrecher, C. Hipp, and D. Kortschak. Higher-order expansions for compound distributions and ruin probabilities with subexponential claims. Scand. Actuar. J., (2):105-135, 2010.

[2] A.V. Asimit and A.L. Badescu. Extremes on the discounted aggregate claims in a time dependent risk model. Scand. Actuar. J., (2):93-104, 2010.

[3] A.V. Asimit and B.L. Jones. Asymptotic tail probabilities for large claims reinsurance of a portfolio of dependent risks. Astin Bull., 38:147-159, 2008.

[4] A.V. Asimit and B.L. Jones. Dependence and the asymptotic behavior of large claims reinsurance. Insurance Math. Econom., 43(3):407-411, 2008.

[5] V.A. Asimit, E. Hashorva, and D. Kortschak. Asymptotic tail probability of randomly weighted large risks. Submitted, 2013.

[6] P. Barbe and W.P. McCormick. Asymptotic expansions of convolutions of regularly varying distributions. J. Aust. Math. Soc., 78(3):339-371, 2005.

[7] P. Barbe and W.P. McCormick. Asymptotic expansions for infinite weighted convolutions of heavy tail distributions and applications. Mem. Amer. Math. Soc., 197(922):viii+117, 2009.

[8] J. Beirlant and J.L. Teugels. Limit distributions for compounded sums of extreme order statistics. J. Appl. Probab., 29(3):557-574, 1992.

[9] L. de Haan and A. Ferreira. Extreme Value Theory. Springer, New York, 2006.

[10] M. Degen, D.D. Lambrigger, and J. Segers. Risk concentration and diversification: second-order properties. Insurance Math. Econom., 46(3):541-546, 2010.

[11] M. Denuit, J. Dhaene, M. Goovaerts and R. Kaas. Actuarial Theory for Dependent Risks. John Wiley, England, 2005.

[12] G. Draisma, L. de Haan, L. Peng, and T.T. Pereira. A bootstrap-based method to achieve optimality in estimating the extreme-value index. Extremes, 2(4):367-404 (2000), 1999.

[13] J. Geluk, L. de Haan, S. Resnick, and C. Stărică. Second-order regular variation, convolution and the central limit theorem. Stochastic Process. Appl., 69(2):139-159, 1997.

[14] J.L. Geluk, L. Peng, and C.G. de Vries. Convolutions of heavy-tailed random variables and applications to portfolio diversification and MA(1) time series. Adv. in Appl. Probab., 32(4):1011-1026, 2000. 
[15] E. Hashorva, C. Ling, and Z. Peng. Second-order tail asymptotic of deflated risks. Submitted, 2013.

[16] L. Hua and H. Joe. Second order regular variation and conditional tail expectation of multiple risks. Insurance Math. Econom., 49(3):537-546, 2011.

[17] D. Kortschak. Second order tail asymptotics for the sum of dependent, tail-independent regularly varying risks. Extremes, 15(3):353-388, 2012.

[18] E. Kremer. An asymptotic formula for the net premium of some reinsurance treaties. Scand. Actuar. J., (1):11-22, 1984.

[19] S.A. Ladoucette and J.L. Teugels. Analysis of risk measures for reinsurance layers. Insurance Math. Econom., 38(3):630-639, 2006.

[20] S.A. Ladoucette and J.L. Teugels. Reinsurance of large claims. J. Comput. Appl. Math., 186(1):163-190, 2006.

[21] S.A. Ladoucette and J.L. Teugels. Asymptotics for ratios with applications to reinsurance. Methodol. Comput. Appl. Probab., 9(2):225-242, 2007.

[22] T. Mao and T. Hu. Second-order properties of risk concentrations without the condition of asymptotic smoothness. Extremes, 2013, 16, 383-405.

[23] T. Mao, W. Lv, and T. Hu. Second-order expansions of the risk concentration based on CTE. Insurance Math. Econom., 51(2):449-456, 2012.

[24] E. Omey and E. Willekens. Second order behaviour of the tail of a subordinated probability distribution. Stochastic Process. Appl., 21(2):339-353, 1986.

[25] C. M. Ramsay. The distribution of sums of certain i.i.d. Pareto variates. Comm. Statist. Theory Methods, 35(1-3):395-405, 2006.

[26] S. I. Resnick. Extreme values, regular variation, and point processes. Springer-Verlag, New York, 1987. 